\title{
Cognitive Self-Scheduled Mechanism for Access Control in Noisy Vehicular Ad Hoc Networks
}

\author{
Mario Manzano, ${ }^{1}$ Felipe Espinosa, ${ }^{1}$ Ángel M. Bravo-Santos, ${ }^{2}$ and Alfredo Gardel-Vicente ${ }^{1}$ \\ ${ }^{1}$ Electronics Department, Polytechnic School, University of Alcala, Campus Universitario s/n, 28871 Alcalá de Henares, Spain \\ ${ }^{2}$ Signal and Communications Theory Department, Higher Polytechnic School, Carlos III University, \\ Avenida de la Universidad 30, 28911 Leganes, Spain
}

Correspondence should be addressed to Felipe Espinosa; espinosa@depeca.uah.es

Received 15 August 2014; Accepted 17 November 2014

Academic Editor: Jinhui Zhang

Copyright (C) 2015 Mario Manzano et al. This is an open access article distributed under the Creative Commons Attribution License, which permits unrestricted use, distribution, and reproduction in any medium, provided the original work is properly cited.

\begin{abstract}
Within the challenging environment of intelligent transportation systems (ITS), networked control systems such as platooning guidance of autonomous vehicles require innovative mechanisms to provide real-time communications. Although several proposals are currently under discussion, the design of a rapid, efficient, flexible, and reliable medium access control mechanism which meets the specific constraints of such real-time communications applications remains unsolved in this highly dynamic environment. However, cognitive radio (CR) combines the capacity to sense the radio spectrum with the flexibility to adapt to transmission parameters in order to maximize system performance and has thus become an effective approach for the design of dynamic spectrum access (DSA) mechanisms. This paper presents the enhanced noncooperative cognitive division multiple access (ENCCMA) proposal combining time division multiple access (TDMA) and frequency division multiple access (FDMA) schemes with CR techniques to obtain a mechanism fulfilling the requirements of real-time communications. The analysis presented here considers the IEEE WAVE and 802.11p as reference standards; however, the proposed medium access control (MAC) mechanism can be adapted to operate on the physical layer of different standards. The mechanism also offers the advantage of avoiding signaling, thus enhancing system autonomy as well as behavior in adverse scenarios.
\end{abstract}

\section{Introduction}

The scientific community has shown an increasing interest in intelligent transportation systems (ITS) over the last few years. Early attempts to apply information technologies to road transport systems yielded various approaches, such as digital short range communications (DSRC) [1] in the USA. Later, with the goal of establishing a universal framework, several international bodies launched their proposals to support future intelligent transport system applications [2]. These agencies include an industrial consortium, the car to car communication consortium (C2C-CC) [3], the international organization for standardization (ISO), which has developed the communications access for land mobiles (CALM) initiative, the CVIS (cooperative vehicle infrastructure systems) project $[4,5]$, and the IEEE, which has developed the wireless access for the vehicular environment (WAVE) protocol suite [6] and the 802.11p specification [7], which is considered the most acceptable proposal of all these frameworks.

More recently, several technology companies, research centers, and leading vehicle manufacturers have been developing a number of alternatives in order to offer vehicles with different automatic driving levels [8-10]. Several research bodies and technology companies have demonstrated their proposals, including VisLab [11], IAI-CSIC, which has developed the AUTOPIA project [12], Google [13], and Bosch [14]. Consequently, ITS are considered a very important source of innovative ideas, projects, and applications, for example, those related to platooning guidance $[15,16]$ or others related to more typical situations in ITS $[17,18]$.

One of the most interesting challenges in regard to these systems is to develop a universal wireless communication system which provides the communication capacity required by the vast majority of possible applications envisaged for 
this field. Such is the aim of the IEEE standard known as WAVE [6], which includes the 802.11 p specification [7], a new member of the extensive IEEE 802.11 family. The design of this new specification is based on the great advantages of the other protocols in the 802.11 family but also includes particular modifications to improve its adaptation to vehicle surroundings $[6,7]$.

The IEEE 802.11p medium access control protocol incorporates the carrier sensing multiple access/collision avoidance (CSMA/CA) mechanism contained in the $b$ and $g$ specifications widely used to gain access to networks and the Internet. In order to improve system performance, enhanced distributed channel access (EDCA), an update in the IEEE 802.11a specification, has been included to provide quality of service [19]. However, not all ITS applications have the same communication requirements. Furthermore, some traffic safety applications require real-time communication with high reliability, meaning that packets must be successfully delivered before a certain deadline [20-23]. To meet a realtime deadline, timely and predictable access to the channel is paramount; however, the medium access method used in IEEE 802.11p does not guarantee channel access before a finite deadline [21-23].

Several techniques within the cognitive radio (CR) paradigm [24-26] have recently been developed related to spectrum exploration [27-29] and dynamic spectrum access [30-32]. These techniques can be applied to the design of medium access control mechanisms in the quest for solutions offering a better performance than the conventional proposals.

This paper outlines the enhanced noncooperative cognitive multiple access (ENCCMA) proposal, which has evolved from the noncooperative cognitive time division multiple access (NCC-TDMA) proposal [20,33]. Dynamic spectrum access (DSA) and spectrum exploration techniques are used in order to expand the mechanism's capacities and improve its performance by applying a combination of both time division multiple access (TDMA) and frequency division multiple access (FDMA) schemes. The rest of the paper is organized as follows. Section 2 gives a detailed description of the problem. Section 3 presents an analysis of recent papers on cognitive radio. Section 4 provides a description of the model of the system analyzed. The ENCCMA proposal is described in detail in Section 5, while the simulation results and conclusions are given in Sections 6 and 7, respectively.

\section{Real-Time Communication in ITS}

The physical layer and MAC sublayer of the WAVE standard developed by the IEEE to meet ITS requirements are described in the IEEE 802.11p specification [7].

Various attempts have been made in the design of the WAVE medium access control sublayer to simplify the connection and information exchange operations in local area wireless networks and to provide effective use of ad hoc communication among vehicles [6]. Consequently, a new communication mode, the WAVE mode, has been included in the specification. This mode does not require prior network authentication and connection, which therefore reduces the time and signaling required for a node to send information to network members.

However, as with the rest of the IEEE 802.11 family, the IEEE 802.11p uses CSMA/CA as a medium access control (MAC) mechanism. In addition, IEEE 802.11p also includes enhanced distributed channel access (EDCA) in order to improve the performance of this mechanism in ITS scenarios [19]. This mechanism was already included in the IEEE 802.11e amendment to improve quality of service by classifying traffic according to four different priorities. Therefore, the resulting medium access control mechanism is contention-based, rather than deterministic, since it cannot guarantee medium access within a bounded time [21], and thus the improvement provided by EDCA does not ensure deterministic access delay. EDCA performance depends on the level of priority assigned to messages exchanged within the system $[19,34]$, and the existence of a large number of high priority messages will reduce effectiveness. Because of this nondeterministic nature of the IEEE 802.11p, it is not suitable for traffic safety applications where low-delay, reliable, realtime communication is required.

There are several published solutions that attempt to improve the properties of the IEEE 802.11p medium access control mechanism and satisfy the specific functions required by ITS applications. These are classified as contention-based and schedule-based protocols [35]. Schedule-based dynamic schemes are suitable for networks in which the topology or members are variable. In these cases, access schedules are continuously calculated to reflect network changes. Selforganized dynamic access is more suited to the special characteristics of vehicular environments [20]. In this case, allocation becomes the responsibility of the system users themselves. Consequently, the medium access control mechanism can contend with high levels of user mobility and inherent network topology variations in ITS.

Another possible classification for MAC mechanisms in the field of ITS can be made between proposals for a final specific application in the system versus fully generic solutions whose operation is not restricted to a certain type of application. There are several proposals for applicationbased MACs, such as position-based MACs which leverage the need to disseminate the current position, speed, and direction of vehicles in the context of ITS. The self-organizing time division multiple access (STDMA) mechanism [21] is an illustrative example of this type of proposal, which requires the transmission of protocol signaling in order to allow users to generate access planning.

This paper presents a generic, self-organized, and scheduled medium access control mechanism. This proposal provides the deterministic features required by real-time communications in the ITS field. Moreover, it can be adapted to any application and act as a single MAC or as a complementary MAC for the solution.

\section{Cognitive Radio}

This section describes how cognitive radio has become an important source of new techniques frequently used to design medium access control mechanisms. Several proposals in 
this field have recently leveraged both spectrum sensing and dynamic spectrum access, including the present ENCCMA proposal.

3.1. Cognitive Radio Properties. Cognitive radio [24-26] is an intelligent wireless communication system that is aware of its environment and uses understanding-by-building methodology to learn from the environment and adapt to statistical variations in the input stimuli by effecting the corresponding changes in determined real-time parameters. The twofold aim of CR, to obtain a highly reliable and efficient communication system, constitutes a perfect match with ITS requirements.

The core of the cognitive network is the cognitive cycle [36], which consists of six processes: observe, orient, plan, decide, act, and learn. A cognitive network achieves networklevel cognition by integrating the cognitive cycle across layers in the protocol stack and through the network nodes. Such cognitive nodes require an architecture that supports observation of the state of the network, collective reasoning to achieve end-to-end network goals, learning from past actions, and reconfiguration of cognitive nodes based on collective decisions.

One of the main ideas that CR exploits is observation of spectrum use in order to take advantage of free spectrum zones (spectrum holes) at any given moment. Searching for these holes and using them constitute one of the main objectives of CR [37]. Consequently, CR systems have receptors observing the medium (spectrum sensing) [27] and detecting occupancy by any kind of signal.

The result of this spectrum occupancy observation leads to one of the greatest challenges of CR-based systems: the design of dynamic spectrum access techniques. These techniques are designed according to the level of cooperation among users and range from noncooperative distributed schemes to completely centralized ones.

3.2. Spectrum Sensing and Dynamic Spectrum Sensing. In a previous paper presenting the NCC-TDMA proposal $[20,33]$, the concepts of CR were applied in terms of spectrum sensing, learning, and system adaptability to the radio environment.

Observation of medium occupancy is one of the most important capabilities of CR, as this enables detection of underutilized spectrum holes which can therefore be used for transmissions by secondary users. This feature of CR is associated with spectrum sensing and DSA techniques, with the latter being defined as a method to adapt to the use of spectrum resources to the present situation and user requirements [30]. Both of them now constitute one of the main pillars in the design of medium access mechanisms, as can be seen in $[27,31]$.

One example of a medium access control mechanism using these techniques is cognitive MAC for VANET (CMV) [38]. In this paper, the spectrum sensing techniques described in [28] are applied over the channel distribution described in the WAVE standard.

3.3. Multichannel in WAVE. Following the guidelines established in DSRC [1], the WAVE standard includes a multichannel operation based on seven different channels: six of them are called service channels ( $\mathrm{SCH})[34,39]$, while the seventh is the control channel $(\mathrm{CCH})$ [6]. Although the EDCA strategy is applied to each of these channels, the control one is responsible for critical messages, or, in terms of ITS, real-time communications. Moreover, the possibility is provided of using a higher transmission power for the control channel in order to improve the likelihood of successfully carrying out a transmission on that channel. In summary, the capacity of WAVE to support real-time communications can be measured by the operation of the protocol on the control channel.

3.4. Learning and Adaptability. The NCC-TDMA proposal leverages the concept of learning and adaptability through the estimation allocation vector (EAV). This tool enables each user to associate each transmission slot to a value of the estimated probability of finding that slot free for transmission. The values of this vector are updated in accordance with the result of the previous transmission attempt using the algorithm described in [20]. This same idea is applied in the CMV mechanism [38]. Unlike the generic approach employed in NCC-TDMA, CMV specifically executes the mechanism over the WAVE channel scheme using a tool named spectrum status table (SST), which stores the status of the spectrum sensed near the device. By storing this information, each user can adapt to any environment condition, at least to those registered in the SST in the case of CMV [38] or in the EAV in the case of NCC-TDMA.

The advantages of this mechanism are taken to a higher level in the proposal described in the present paper. The ENCCMA exploits this functionality and enhances it in two ways:

(i) by maintaining a generic approach that is not restricted to the division of channels included in WAVE but is rather applied to a division of both frequency channels and time slots using a combination of TDMA and FDMA and the estimation allocation vector (EAV) thus becomes the estimation allocation matrix (EAM);

(ii) by improving the accuracy of the information stored for each user through the inclusion of a secondary updating mechanism that decreases the time required for the information stored in the EAM to accurately reflect spectrum occupancy conditions at any given time.

The next section describes the model of the system designed, analyzed, and simulated in order to develop the ENCCMA proposal described in detail in Section 5.

\section{System Model}

The starting point for an analysis of the ENCCMA proposal is platooning guidance of autonomous vehicles. In this collaborative transport scenario, vehicles are grouped into convoys, and each convoy is headed by a leader vehicle which transmits the route information to the rest of the convoy members 
(followers). Control of each follower requires knowledge of the motion state of the preceding unit, and the dissemination of this information is critical because loss of these messages could jeopardize the stability of the convoy chain [15]. In addition, other maneuvers can produce variations in spectrum occupancy, such as vehicles joining or leaving the convoy. In summary, it is a system in which users must convey critical information within a specified maximum deadline and the system itself must cope with a variable number of users as well as different interfering signals due to the existence of other convoys or other services sharing the medium.

4.1. Scenario. To simplify the experiments, the special conditions of start, stop, line change, and the generation of reference paths for the convoy on a real map are not discussed here. Thus, the convoy can be considered as a group of freely flowing vehicles on an unbounded map. It is assumed that the vehicles are traveling in convoy and can separate from it (leaving the communication system) in the same way that other vehicles can join it as new users.

Trajectory control and tracking tasks require cooperation among users. However, from the point of view of the communication system and CR, the system is not cooperative because the messages exchanged among users only contain application information which is not related to management of the medium access control mechanism. There is no need for signaling among users as the vehicles never send information about the transmission region they are using. This feature renders users completely autonomous from the communication point of view.

4.2. Channel. According to the definition of WAVE, the shared medium should be divided in accordance with the channel scheme included in the specification [6]. As a result, only the seven specified channels may be applied, ignoring the specific use defined in the standard for each channel. It should be noted that, for the simulation experiments conducted, the shared medium was divided into channels in a generic way in order to avoid the temporary restriction imposed by the WAVE specification. The main requirement of real-time applications in ITS is that the exchange of messages must be made within a critical time limit $T_{c}$. The shared medium is divided according to this limit in order to conform to the TDMA-FDMA frame. Both time slot duration and channel frequency range are determined by the needs of the application.

In addition, the following is assumed.

(a) During each transmission, it is possible to transmit all the information needed by each user to fulfill the system requirements.

(b) Once the medium has been accessed, there are no external interferences in the communication process.

4.3. Communication. To perform the envisaged functions of the new proposal, it is assumed that all users rely on a communication system capable of observing the medium (spectrum sensing) in the same way as the systems described in [2729], which is equipped with an auxiliary device providing time synchronization [27]. Given both these capabilities, the end users will be able to manage their communications themselves in a decentralized and autonomous way without the need to receive information from any other user in the system (signaling).

4.4. Key Aspects. In summary, the following aspects characterize the model of the communication system under study.

(i) The shared medium is exploited using a combination of TDMA and FDMA schemes. The main frame lasts $T_{c}$ and is divided into $r$ time slots. Each time slot is also divided into $f$ frequency channels.

(ii) Thus, a total number of $r f$ transmission regions are established for the system users in a multiframe.

(iii) Each participant must transmit his data within the time limit $T_{c}$. Thus, it is necessary and sufficient to access the medium in one transmission region.

(iv) Once a participant has accessed the medium during the period of transmission in the selected region, it will be considered that the transmission has been carried out correctly.

(v) Vehicles are equipped with a spectrum sensing communication system which provides each user with spectrum occupancy knowledge.

(vi) Vehicles are equipped with time devices to provide multiframe synchronization.

(vii) For the medium access control mechanism, there is no cooperation among users. They neither exchange signaling nor have explicit knowledge of transmission regions used by the remaining users.

\section{The ENCCMA Proposal}

The enhanced noncooperative cognitive medium access (ENCCMA) proposal represents a development of the NCCTDMA mechanism which improves the latter's efficiency by guaranteeing a bounded medium access delay to ITS applications which require real-time communications.

The original proposal consisted of a TDMA-based medium access control mechanism. Each user stored information about the occupancy estimation of each slot by applying CR techniques. This information was then used to select the best slot to perform the information transmission required by the application. Subsequently, that information was updated depending on the result of each of these transmissions by applying a bonuses-and-penalties algorithm. This was a nondeterministic mechanism that enabled each user to learn and adapt to rapid changes in VANET environments.

The ENCCMA mechanism has been designed in response to the need for an improvement in the application of DSA and $\mathrm{CR}$ techniques to enhance the performance of the original proposal. 
5.1. Increasing System Capacity. As with other existing alternatives [38], including WAVE itself [6], the ENCCMA proposal incorporates a multichannel operation. Following the principle of neutrality and generality of the mechanism, it is not applied in a fixed frequency division scheme. Rather, the mechanism has been designed bearing in mind that the availability of spectrum and channels can be freely set. Therefore, the shared medium is not only divided into time slots, as in the NCC-TDMA, but an FDMA scheme is also implemented in each time slot. Thus, the medium is operated using a combination of TDMA and FDMA schemes, resulting in transmission regions defined by time slots and frequency channels which notably increase the maximum number of users that can interact in a given application. The granularity of these divisions is not restricted by the mechanism but rather the requirements of the application determine the medium division scheme.

The tool used by the NCC-TDMA mechanism to store the estimated spectrum occupancy information was a vector whose elements represented the estimated occupancy information for each user and time slot. In the ENCCMA proposal and with the emergence of multichannel operations, this vector has evolved into a matrix, the estimation allocation matrix (EAM), in which each user can store his information about the estimated occupancy of each transmission region. As with the NCC-TDMA, it is necessary to organize the access to the multiframe transmission regions according to the possibility of finding these regions occupied by other system users. Consequently, the use of transmission schemes operates as shown in the example illustrated in Figure 3. It should be noted that this operation is performed while maintaining the constraint of avoiding signal transmission, rendering each user completely autonomous in the system.

5.2. Improving Spectrum Sensing. The ENCCMA strategy provides a substantial improvement in spectrum scan capabilities compared to the original NCC-TDMA proposal. The estimated occupancy information stored in the EAM changes as a result of accessing the medium to perform the transmission required by the application. In addition, it also changes by means of a complementary exploration mechanism, the aim of which is to increase the accuracy of the stored information within the shortest possible time. Therefore, users can select the best transmission region in each iteration. However, this additional exploration is not free in terms of the efficiency pursued by CR. Therefore, the ENCCMA strategy establishes a cost function in order to determine the optimal number of regions to be explored in each iteration. This cost function can be adapted to the needs of each application or system by modifying the parameters shown in Table 1.

5.3. Performance Index. A performance index has been designed with the aim of increasing the adaptability, learning, and efficiency of the system. It describes the exploration cost in the system and it allows the algorithm to determine the optimum number of regions to inspect in each iteration.
TABLE 1: Parameters used to obtain the cost function curve of Figure 1.

\begin{tabular}{lc}
\hline Parameter & Value \\
\hline Total number of available transmission regions & 100 \\
Number of previous iterations $(N$ value in $(5))$ & 20 \\
Number of regions explored in previous iterations & 50 \\
Unit cost of scan & From 0.5 to 2 \\
$s$ & 10 \\
$\alpha$ & 0.9 \\
$d_{s}$ & 10 \\
$w$ & 0.7 \\
\hline
\end{tabular}

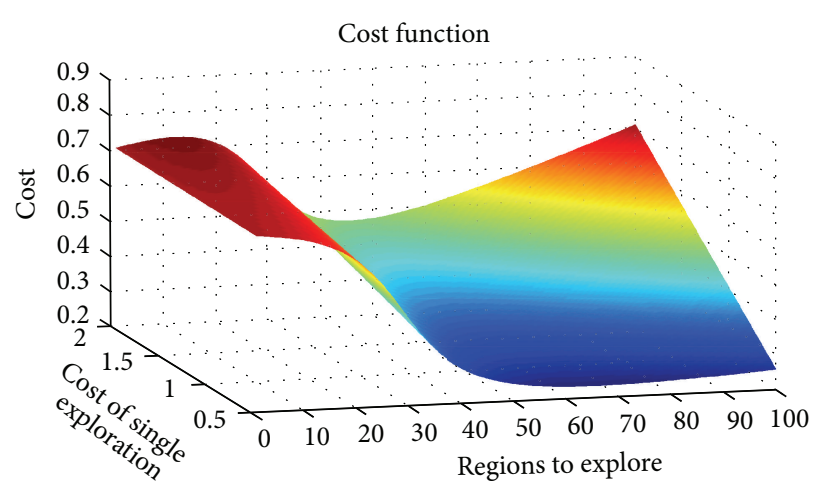

FIGURE 1: Cost function: cost of exploration versus regions to explore.

To take into account all the elements of the system, in the design process the following considerations have been made.

(1) Resources Consumption versus Spectrum Occupancy Knowledge. Two main terms determine the algorithm decision: the cost of exploring the regions and the current knowledge of the spectrum occupancy. It is necessary to maintain a compromise solution between the algorithm performance (always exploring everything in the ideal case) and the minimum consumption situation (not to explore any extra region).

The parameter $W$ was included to balance the relative importance of each term in the performance index evaluation:

$$
C=W \cdot E+(1-W) \cdot R
$$

where:

(i) $C$ is cost function or performance index,

(ii) $W$ is weight parameter, aimed to balance the importance of the two main terms in the performance index; $W \in(0,1)$,

(iii) $E$ is parameter related to the current spectrum occupancy knowledge, and

(iv) $R$ is parameter related to the cost of exploration.

(2) Parameter Related to the Cost of Exploration. The definition of the term $R$ implies, on one hand, a relation between 


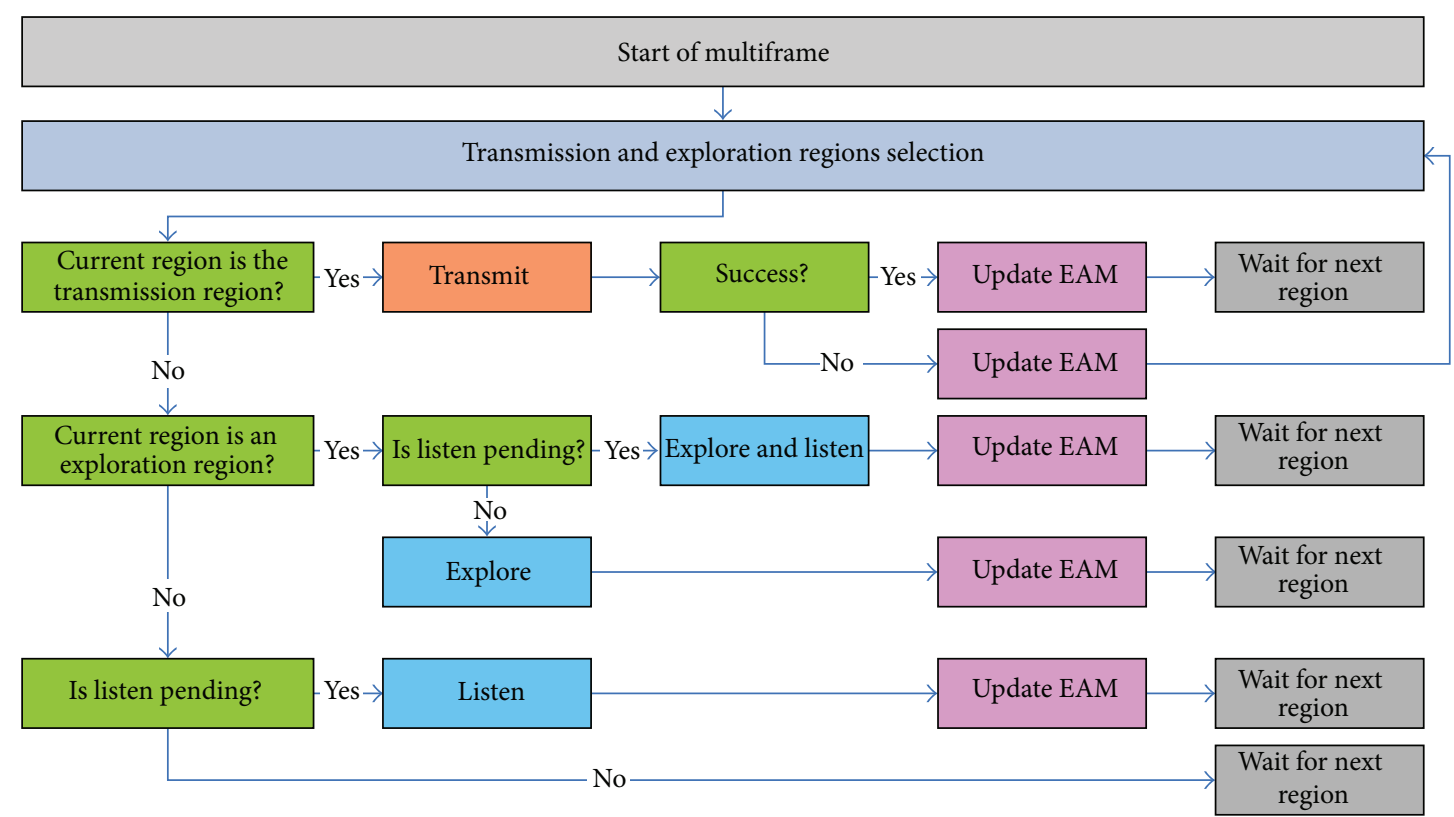

FIGURE 2: ENCCMA algorithm flowchart.

Vehicle 2

\begin{tabular}{|c|c|c|c|c|c|}
\hline Channel 4 & \multicolumn{3}{|c|}{ Listen-no rx. } & Listen-no rx. & Exploration \\
\hline Channel 3 & Tx. & Tx. & Check & Listen-no rx. & \\
\hline Channel 2 & \multicolumn{3}{|c|}{ Listen-no rx. } & Listen-rx. ok & \\
\hline Channel 1 & \multicolumn{3}{|c|}{ Listen-no rx. } & Listen-no rx. & \\
\hline & \multicolumn{3}{|c|}{ Slot 1} & Slot 2 & Slot 3 \\
\hline
\end{tabular}

Vehicle 3

\begin{tabular}{|c|c|c|c|c|c|}
\hline Channel 4 & Listen-no rx. & & & & \\
\hline Channel 3 & Listen-rx. ok & & Check & Tx. & Tx. \\
\hline Channel 2 & Listen-no rx. & & \multicolumn{3}{|c|}{ Exploration } \\
\hline Channel 1 & Listen-no rx. & Exploration & & & \\
\hline
\end{tabular}

Example of medium occupancy in ENCCMA

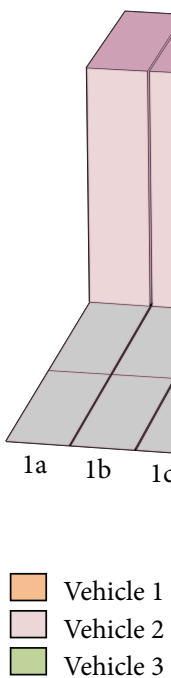

FIGURE 3: Application of TDMA-FDMA multiframe and transmission schemes in ENCCMA to an example of two followers and a leader formation. Three time slots and four frequency channels are available for the MAC mechanism.

the number of regions to explore in the iteration and the total number of regions and, on the other hand, the cost of exploration of a single region which is a term defined by the hardware characteristic of the system. The number of regions to explore is the value that the algorithm has to determine by minimizing the cost function. Therefore,

$$
R=\frac{X}{T} R_{c},
$$

where

(i) $X$ is number of regions to explore, (ii) $T$ is total number of regions, and

(iii) $R_{c}$ is exploration cost of a single region.

(3) Parameter Related to the Spectrum Occupancy Knowledge. The parameter $E$ is defined from a curve obtained from a set of configuration parameters and a variable related to the knowledge of the historical spectrum occupancy. If the information about spectrum occupancy is recent, the number of regions to explore can be reduced. However, if the information was obtained many iterations ago, the number 
of explorations must be increased. The configuration parameters $s$ and $d$ are considered for allowing the adaptation of the performance index to different scenarios. The parameter $s$ is simply a slope modifier that can be used to soften the curve variation near the minimum. On the other hand, the parameter $d$ contains the information about the exploration in previous iterations. Other variables have been included in the definition of $E$ in order to constrain its value in the range $(0,1)$. The resulting expression for $E$ is

$$
E=1-\left(\frac{1}{\pi} a \tan \left(\frac{d-T+2 X}{2 s}\right)+a \tan \left(\frac{T-d}{2 s}\right)\right),
$$

where $s \in(1,30)$ and $d$ includes the information about the exploration history.

The term $d$ represents a measure of the previous knowledge about the spectrum occupancy. It depends on the total number of regions $T$, a configuration parameter $d_{s}$, and the factor $h$ which contains the information about the previous iterations:

$$
d=d_{s} T h
$$

The factor $h$ is evaluated as a sum of $N$ values that represent the number of explorations $X_{n}$ performed at iteration $n$, normalized by the total number of regions $T$, and weighted by an exponential function with an adjusting parameter $\alpha$ $(\alpha \in(0,1))$, as shown in the following equation:

$$
h=\sum_{n=1}^{n=N} \frac{X_{n}}{T} e^{\alpha(n-N)}
$$

According to this definition, the number of regions explored in a recent iteration will be more relevant for $h$ than the number of regions explored in a previous iteration. Therefore, a higher value of $h$ will determine a lower value of the number of regions to explore. In the same way, the higher the value of the parameter $\alpha$ is, the lower the value of $h$ is. It means that if in the previous iterations there were not a large number of explorations, in the current iteration the vehicle needs to perform a lot of them to reach a certain level of spectrum occupancy knowledge.

The performance index $C$ is completely defined by expressions (1) to (5). The algorithm determines the number $X$ of regions to explore in each iteration by obtaining the minimum $C$ value.

In Table 1, the values of parameters used to obtain the cost function plotted in Figure 1 are shown. The same values are used in the simulations performed in Section 6.

As the parameter $d$ is related to the exploration history and depends on the number of regions explored in each iteration, the resultant performance index is time dependent and therefore the algorithm has to find the minimum value of the curve in each iteration.

5.4. The ENCCMA Algorithm. The ENCCMA algorithm manages the actions of each user in each iteration. Basically, it determines the transmission region and the exploration regions (number and identification). It also updates the
EAM with the information obtained from the explorations. The following actions can be performed by each user when accessing the medium: transmit, listen, and explore and listen. The transmit action is performed in the transmission region determined by the algorithm for that purpose. The listen action is performed in order to receive the information transmitted by the other system users. Besides receiving information provided by other users, the explore and listen action senses the medium in the regions identified by the algorithm for this purpose without interfering with any other potential users transmitting in the same regions. Figure 2 presents a flowchart of the ENCCMA algorithm.

5.5. Transmission Schemes. According to the ENCCMA transmission mechanism, each transmission region is divided into three time intervals and each user makes use of them in a different way. If the selected region is the same as the one in the previous iteration, the listen + transmission scheme is applied. First, the user transmits the required information and listens during the third interval of the region to check for the presence of any interference. If the selected region is different from the one selected in the previous iteration, the user senses the medium to check the availability of this region. If it is free, the user transmits its information; otherwise, the user has to reexecute the algorithm to check another region.

An example of the application of different transmission schemes can be seen in Figure 3, where a convoy of three units exchanges motion information to guarantee the stable movement of the formation. The medium access control mechanism considers three time slots and four frequency channels.

5.6. Estimation Allocation Matrix Update. The EAM is the tool that enables each user to determine the present status of the medium. In the ENCCMA, the EAM is rapidly updated via the auxiliary scanning process. Before transmission, each element of the matrix represents an estimate of finding the associated transmission region unoccupied. Each scan of the available transmission regions provides occupancy information which is used by the algorithm to apply bonuses if the region is found to be free or penalties if it is found to be busy. Thus, a penalty is applied to a matrix element associated with an occupied region, in order to lower its possibilities of being selected as a transmission region by the algorithm. In contrast, a bonus is applied to a matrix element associated with a region found to be unoccupied in each iteration, facilitating its selection as transmission region for the user in future iterations. A detailed definition of the bonuses and penalties used for the EAM update can be found in $[20,33]$. A user who has successfully transmitted, after applying bonuses, remains in the listen state until the next frame; otherwise, a new iteration is triggered.

5.7. Summary of Main Features. Summing up, the main aspects of the ENCCMA proposal are as follows.

(i) It represents a natural evolution of the NCC-TDMA strategy described in [20]. 
(ii) Improvements have been made in two main areas:

(a) leverage of multichannel capacity, increasing the system's capacity by using a combination of TDMA and FDMA schemes;

(b) leverage of new CR techniques in the field of spectrum sensing [27] and dynamic spectrum access [30]. Consequently, this new proposal senses medium status (spectrum occupancy) and reflects potential environment variations in VANET scenarios more rapidly. It allows each user to select the best region to transmit in each iteration.

(iii) These original restrictions have also been considered.

(a) The resultant MAC must meet the real-time communications requirements of VANET scenarios.

(b) The use of signaling must be avoided in order to maintain the complete autonomy of each system user. Each user must be able to perform any application function by transmitting the required information without the need to receive any signaling information from any other user or central station.

(iv) A generic approach has been employed in the design of the ENCCMA.

(a) The protocol can be used as the main medium access control mechanism or as a complementary one.

(b) The new multichannel capacity can be freely adapted to any spectrum division scheme, including the IEEE 802.11p multichannel scheme or any other multichannel configurations.

(v) Various aspects of the cost function defined for the complementary exploration function can be configured:

(a) depending on the cost of a single operation of spectrum sensing in terms of energy efficiency;

(b) the balance between historical exploration values and immediate ones.

\section{Results}

To evaluate the performance of the ENCCMA, a specific tool called SIMITS was implemented using NET C\# (available in [40]). This tool starts from a scenario that matches the characteristics described in Section 4 and allows the user to select the following main aspects: number of users, time as well as frequency divisions, and medium access control mechanisms, including Slotted-Aloha [41], RR-ALOHA [42], and the ENCCMA proposal presented here. Slotted-Aloha is one of the most widely studied protocols in the literature. This generic MAC mechanism is not oriented to the application and does not use signaling. RR-ALOHA is based on SlottedAloha but includes a signaling mechanism to render the mechanism self-scheduled.

Section 3 made a reference to the cognitive MAC for VANET (CMV). However, the SIMITS tool does not include this alternative among its available protocols because it is a specific mechanism for the spectrum division given in IEEE 802.11p. This means that it is not possible to perform a simulation using CMV over a generic medium scheme without modifying its performance. It should be noted that both Slotted-Aloha and RR-ALOHA are proposals which start from a TDMA scheme. However, SIMITS is capable of simulating environments with combined TDMA and FDMA schemes for these access mechanisms without taking into account the considerations of a real implementation of these protocols over any kind of combined TDMA and FDMA schemes. This is also the reason why all the selected mechanisms are generic and not application oriented. This means that every MAC selectable by the simulator can operate over any kind of access (TDMA, FDMA, or combinations of TDMA and FDMA). In the case of the previously described protocol (CMV), this is not possible because its nature is strictly associated with the WAVE spectrum division scheme.

In addition, this tool is capable of simulating channel occupancy by means of interfering signals. Therefore, scenarios with very dense spectrum occupancy have been tested, revealing some interesting conclusions about mechanism performance in such types of situation, as can be seen in Section 6.2.

6.1. Throughput Comparison in Nominal Scenario. The software tool calculates the performance of the medium access control mechanism in terms of throughput as follows:

simulated_time $=$ simulated_time_slots $\cdot$ time_per_slot

bytes_sent $=$ successful_transmissions $\cdot$ message_size

$$
\begin{aligned}
\text { bytes_sent_per_user } & =\frac{\text { bytes_sent }}{\text { number_of_users }} \\
\text { total_throughput } & =\frac{\text { bytes_sent } \cdot 8}{\text { simulated_time }} \\
\text { throughput_per_channel } & =\frac{\text { total_throughput }}{\text { number_of_frequency_channels }},
\end{aligned}
$$

where message size is calculated according to the encoding for each modulation type [7], as shown in Table 2.

In the case of RR-ALOHA, it is necessary to take into account the extra size of the message related to the signaling required for the protocol [42]. Consequently, to calculate the throughput when using RR-ALOHA, an extrasized user message is added according to

rr_aloha_user_data_size $=$ user_data $+2 *$ number_of_users. 
TABLE 2: Coding rates used for each modulation in IEEE 802.11p.

\begin{tabular}{lcc}
\hline Modulation & Coding rate & Transfer rate \\
\hline BPSK & $1 / 2$ & $3 \mathrm{Mbps}$ \\
BPSK & $3 / 4$ & $4.5 \mathrm{Mbps}$ \\
QPSK & $1 / 2$ & $6 \mathrm{Mbps}$ \\
QPSK & $3 / 4$ & $9 \mathrm{Mbps}$ \\
16-QAM & $1 / 2$ & $12 \mathrm{Mbps}$ \\
16-QAM & $3 / 4$ & $18 \mathrm{Mbps}$ \\
64-QAM & $2 / 3$ & $24 \mathrm{Mbps}$ \\
64-QAM & $3 / 4$ & $27 \mathrm{Mbps}$ \\
\hline
\end{tabular}

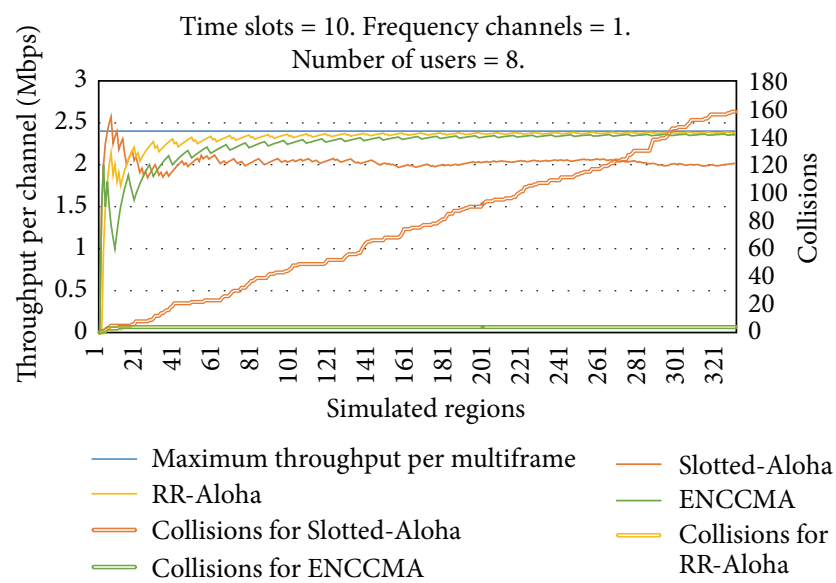

FIgURE 4: Throughput comparison of several MAC mechanisms in an ideal small scenario.

Figure 4 shows the simulation results for a small scenario in ideal conditions (no interferences), where all the mechanisms tended to reach the maximum throughput per user and per frequency channel. As can be seen, both the ENCCMA and RR-ALOHA were capable of reaching an equilibrium situation and avoiding the occurrence of collisions. However, this was not the case of Slotted-Aloha, where collisions occurred throughout the simulation experiment.

The results shown in Figure 5 were obtained using the same conditions as in the previous experiment but with a shared medium divided into a combined TDMA and FDMA scheme and modifying the protocol behavior of SAloha and RR-ALOHA to allow frequency channels. It can be seen that the performance of the tested mechanisms was quite similar to that obtained with the basic scenario, which only considered time division. As expected, SlottedALOHA offered a lower throughput since vehicles using this mechanism collided when trying to communicate.

6.2. Throughput Comparison in a High Interference Scenario. Simulation results were also obtained for the scenario described in the previous section but considering the existence of multiple interfering signals so that the remaining available transmission regions were equal to the number of users. Each vehicle had to use one of these remaining free regions to transmit without interfering with other vehicles

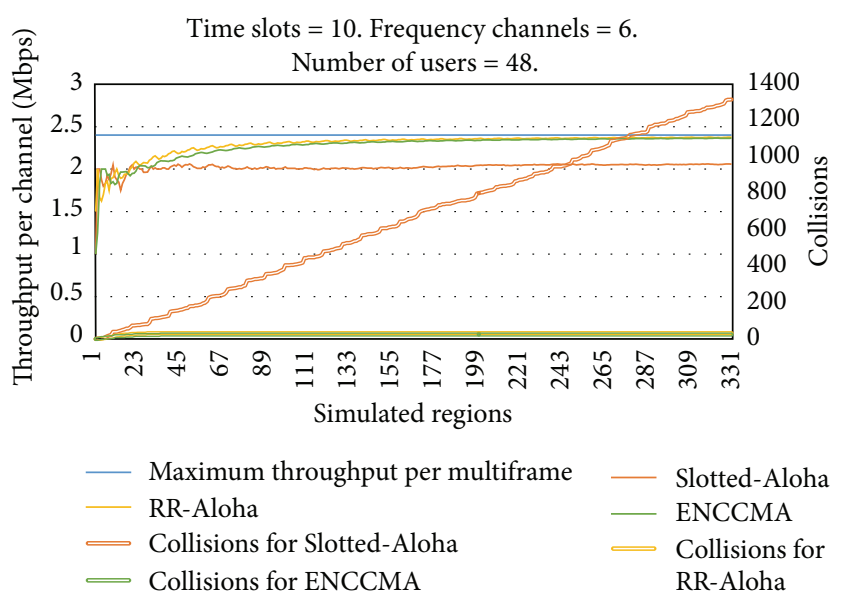

FIgURE 5: Throughput comparison of several MAC mechanisms in an ideal large scenario.

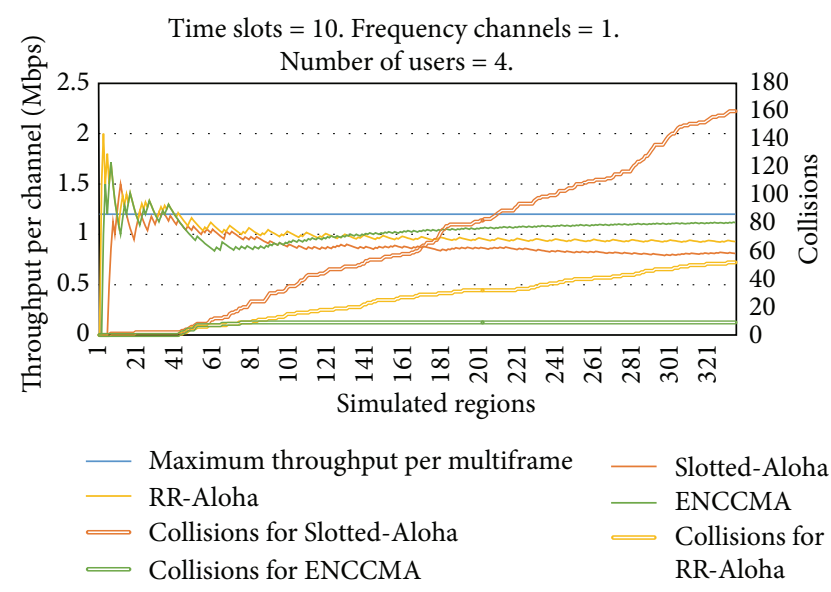

FIGURE 6: Throughput comparison in high interference small scenario.

or with the external signals purposely generated in the experiment. This situation illustrates the capacity of the mechanisms to adapt to changes in the environment and also highlights the capabilities and performance of each evaluated mechanism in similar situations of high interference scenarios.

As can be seen in Figure 6, only the ENCCMA mechanism was capable of recovering equilibrium in the system. In the equilibrium situation, all the users were able to carry out transmission of the information required by the application. In the case of RR-ALOHA, the throughput curve did not reach the downward trend of Slotted-Aloha, but the mechanism was nevertheless still unable to return to the equilibrium situation. For the platooning guidance of autonomous vehicles, only the ENCCMA mechanism could ensure convoy stability. Thus, in order to ensure convoy stability, the system parameters must be configured to maintain the maximum communication time among vehicles lower than the maximum time to reach equilibrium by the medium access control mechanism. It should be noted that the case 


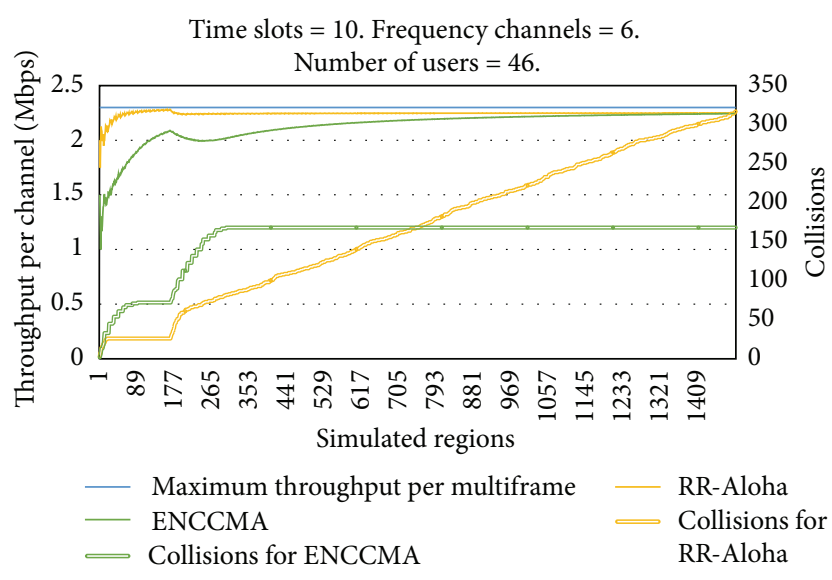

Figure 7: Throughput comparison in high interference large scenario.

under study is a difficult communication scenario due to the presence of multiple interfering signals, which requires the mechanism to work at maximum capacity. In this situation, the mechanism can still reach the equilibrium, but the system is brought to its capacity limit. The same conditions were also applied to a more complex scenario with an increased number of users over a combined TDMA-FDMA scheme, and the simulation results are shown in Figure 7.

The Slotted-Aloha mechanism values have obviously been removed from Figure 7. The behavior of this mechanism was worse than the other two alternatives analyzed, as can be seen in the previous experiments. The RR-ALOHA mechanism reached initial equilibrium faster, due to the benefits that this protocol obtains from using signaling. In this initial stage, the ENCCMA had not yet acquired any knowledge about spectrum occupancy, and thus it required more iterations than RR-ALOHA to reach equilibrium. Given that the medium was divided into 10 time slots and 6 frequency channels in the test scenario, the multiframe system consisted of 60 transmission regions, so that the ENCCMA mechanism reached equilibrium in the second multiframe. Typical values for the specific case of platooning guidance of autonomous vehicles could be about $100 \mathrm{~ms}$. The interferences were introduced into the system around the simulation of transmission region number 180. At this point, both mechanisms had to begin adapting to the shared medium changes in order to recover an equilibrium situation, as required in ITS applications.

The RR-ALOHA mechanism was unable to achieve equilibrium, as indicated by the indefinite increase in the number of collisions. The throughput curve corresponding to this mechanism continued to grow slightly since some users managed to find a transmission region to transmit without collision. However, other users tried to transmit in busy regions, generating collisions and indicating that the mechanism was not able to recover an equilibrium situation.

The ENCCMA mechanism recovered the equilibrium situation following exploration of around 300 regions after two multiframes. This would be around $200 \mathrm{~ms}$, considering typical values in platooning guidance. Although the drop in initial performance of the ENCCMA was more noticeable than in the case of the RR-ALOHA mechanism, it was actually able to recover the equilibrium situation in the worst case scenario. This thus allowed the ITS application to meet the special real-time communication requirements.

\section{Conclusions}

This paper describes an enhanced medium access mechanism which has evolved from the proposal made by the same authors described in $[20,33]$. The aim of the current proposal is to improve the capacity and efficiency of the system by fulfilling real-time communication requirements in environments such as VANET in ITS. In a scenario involving platooning guidance of autonomous vehicles, the loss of critical information endangers the global stability of the convoy formation. This ITS application, in which it is essential to meet real-time requirements, is a clear example of the application of networked control systems to smart transportation.

The ENCCMA proposal can be considered an alternative mechanism for medium access control in VANET applications that require real-time communication. It provides full autonomy to the vehicle in terms of communications as it does not require signaling for operation. In addition, it can be applied to a system as the sole medium access control mechanism or as a complementary one (e.g., as a CSMA/CA complement). Furthermore, it is completely generic and therefore not application oriented. This means that it is not necessary for an application requiring ITS communication among vehicles to meet any special precondition in order to use the mechanism, unlike the case of other proposals such as the STDMA [21], which works better for position dissemination applications. Moreover, it can operate multiple schemes of shared medium division: TDMA, FDMA, or combination of both. It is neither restricted to a particular spectrum division in order to share access among users (as is the case of CMV [38]). Lastly, it is based on the principles of CR (learning and adaptability) and leverages recently developed spectrum sensing and DSA techniques. As a result, it is capable of adapting to rapid changes in medium occupancy either by system users or by external interfering signals.

The global result is a dynamic and autonomous mechanism that takes advantage of medium observation to determine the best transmission region for use each time, and which continuously learns and adapts to environment changes. For guidance of autonomous vehicles in convoy, medium access control is of vital relevance in split and merge maneuvers. In these cases, the medium access control mechanism must deal with new vehicles joining the convoy while maintaining satisfactory transmission for member vehicles. Lastly, although the present version of the mechanism requires time synchronization between system users, a possible future area of research would be to incorporate sufficient complexity into the mechanism to avoid this need.

\section{Conflict of Interests}

The authors declare that there is no conflict of interests regarding the publication of this paper. 


\section{Acknowledgments}

This work has been funded by the Spanish Ministry of Economy and Competitiveness through the ALCOR project (ref. DPI2013-47347-C2-1-R) and by the University of Alcala through the MACRE project (CCG2013/EXP-001).

\section{References}

[1] Y. L. Morgan, "Notes on DSRC \& WAVE standards suite: its architecture, design, and characteristics," IEEE Communications Surveys and Tutorials, vol. 12, no. 4, pp. 504-518, 2010.

[2] S. A. Mohammad, A. Rasheed, and A. Qayyum, "VANET architectures and protocols stacks: a survey," in Communication Technologies for Vehicles, vol. 6596 of Lecture Notes in Computer Science, pp. 95-105, Springer, 2011.

[3] CAR 2 CAR Communication Consortium Manifesto, 2007, http://elib.dlr.de/48380/1/C2C-CC_manifesto_v1.1.pdf.

[4] T. Ernst, V. Nebehaj, and R. Søråsen, "CVIS: CALM proof of concept preliminary results," in Proceedings of the 9th International Conference on Intelligent Transport Systems Telecommunications (ITST '09), pp. 80-85, October 2009.

[5] http://www.cvisproject.org/.

[6] D. Jiang and L. Delgrossi, "EEE 802.11p: towards an international standard for wireless access in vehicular environments," in Proceedings of the 67th IEEE Vehicular Technology Conference (VTC '08), pp. 2036-2040, May 2008.

[7] IEEE 802.11p, "Standard for Information technology-Telecommunications and information exchange between systemsLocal and metropolitan area networks-Specific requirements. Part 11: Wireless LAN Medium Access Control (MAC) and Physical Layer (PHY) Specifications. Amendment 6: Wireless Access in Vehicular Environment".

[8] J. Funke, P. Theodosis, R. Hindiyeh, G. Stanek, K. Kritatakirana, and C. Gerdes, "Up to the limits: autonomous Audi TTS," in Proceedings of the Intelligent Vehicles Symposium, Alcalá de Henares, Spain, June 2012.

[9] http://www.toyota-europe.com/about/news_and_events/ editorial/autonomous-driving.tmex.

[10] https://www.media.volvocars.com/global/en-gb/media/ pressreleases/136182/volvo-car-group-initiates-world-uniqueswedish-pilot-project-with-self-driving-cars-on-public-roads.

[11] A. Broggi, P. Medici, E. Cardarelli, P. Cerri, A. Giacomazzo, and N. Finardi, "Development of the control system for the VisLab intercontinental autonomous challenge," in Proceedings of the 13th International IEEE Conference on Intelligent Transportation Systems (ITSC '10), pp. 635-640, Madeira Island, Portugal, September 2010.

[12] E. Onieva, J. Alonso, J. Pérez, V. Milanés, and T. de Pedro, "Autonomous car fuzzy control modeled by iterative genetic algorithms," in Proceedings of the IEEE International Conference on Fuzzy Systems (FUZZ-IEEE '09), pp. 1615-1620, Jeju Island, Republic of Korea, August 2009.

[13] http://www.google.com/about/careers/lifeatgoogle/selfdriving-car-test-steve-mahan.html.

[14] J. Levinson, J. Askeland, J. Becker et al., "Towards fully autonomous driving: systems and algorithms," in Proceedings of the IEEE Intelligent Vehicles Symposium (IV '11), pp. 163-168, June 2011.

[15] C. Santos, F. Espinosa, D. Pizarro, F. Valdés, E. Santiso, and I. Díaz, "Fuzzy decentralized control for guidance of a convoy of robots in non-linear trajectories," in Proceedings of the IEEE International Conference on Emerging Technologies and Factory Automation (ETFA '10), pp. 1-8, Bilbao, Spain, September 2010.

[16] M. Gerla, E.-K. Lee, G. Pau, and U. Lee, "Internet of vehicles: from intelligent grid to autonomous cars and vehicular clouds," in Proceedings of the IEEE World Forum on Internet of Things (WF-IoT '14), pp. 241-246, March 2014.

[17] M.-W. Li, T.-H. Wu, W.-Y. Lin, K.-C. Lan, C.-M. Chou, and C.H. Hsu, "On the feasibility of using 802.11p for communication of electronic toll collection systems," ISRN Communication and Networking, vol. 2011, Article ID 723814, 11 pages, 2011.

[18] V. Milanés, J. Villagrá, J. Godoy, J. Simó, J. Pérez, and E. Onieva, "An intelligent V2I-based traffic management system," IEEE Transactions on Intelligent Transportation Systems, vol. 13, no. 1, pp. 49-58, 2012.

[19] S. Sehrawat, R. P. Bora, and D. Harihar, "Performance analysis of QoS supported by Enhanced Distributed Channel Access (EDCA) mechanism in IEEE 802.11e," IAENG International Journal of Computer Science, vol. 33, no. 1, 2007.

[20] M. Manzano, F. Espinosa, Á. M. Bravo-Santos, E. Santiso, I. Bravo, and D. Garcia, "Dynamic cognitive self-organized TDMA for medium access control in real-time vehicle to vehicle communications," Mathematical Problems in Engineering, vol. 2013, Article ID 574528, 13 pages, 2013.

[21] K. Bilstrup, E. Uhlemann, E. G. Ström, and U. Bilstrup, "On the ability of the 802.11p MAC method and STDMA to support realtime vehicle-to-vehicle communication," EURASIP Journal on Wireless Communications and Networking, vol. 2009, Article ID 902414, pp. 1687-1472, 2009.

[22] K. Bilstrup, E. Uhlemann, E. G. Ström, and U. Bilstrup, "Evaluation of the IEEE 802.11p MAC method for vehicleto-vehicle communication," in Proceedings of the IEEE 68th Vehicular Technology Conference (VTC '08), pp. 1-5, Calgary, Canada, September 2008.

[23] K. Sjöberg-Bilstrup, E. Uhlemann, and E. G. Ström, “Scalability issues of the MAC methods STDMA and CSMA of IEEE 802.11p when used in VANETs," in Proceedings of the IEEE International Conference on Communications Workshops (ICC '10), pp. 1-5, Capetown, South Africa, May 2010.

[24] S. Haykin, "Cognitive radio: brain-empowered wireless communications," IEEE Journal on Selected Areas in Communications, vol. 23, no. 2, pp. 201-220, 2005.

[25] J. Mitola, Cognitive Radio Architecture: The Engineering Foundations of Radio XML, John Wiley \& Sons, 2006.

[26] E. Hossain and V. K. Bhargava, Cognitive Wireless Communication Networks, Springer, 2007.

[27] D. Cabric, S. M. Mishra, and R. W. Brodersen, "Implementation issues in spectrum sensing for cognitive radios," in Proceedings of the Conference Record of the 38th Asilomar Conference on Signals, Systems and Computers, pp. 772-776, November 2004.

[28] T. A. Weiss and F. K. Jondral, "Spectrum pooling: an innovative strategy for the enhancement of spectrum efficiency," IEEE Communications Magazine, vol. 42, no. 3, pp. S8-S14, 2004.

[29] Z. Yan, Z. Ma, H. Cao, G. Li, and W. Wang, "Spectrum sensing, access and coexistence testbed for cognitive radio using USRP," in Proceedings of the 4th IEEE International Conference on Circuits and Systems for Communications (ICCSC '08), pp. 270274, May 2008.

[30] E. Hossain, D. Niyato, and Z. Han, Dynamic Spectrum Access and Management in Cognitive Radio Networks, Cambridge University Press, Cambridge, UK, 2009. 
[31] Y. Yuan, P. Bahl, R. Chandra, T. Moscibroda, and Y. Wu, "Allocating dynamic time-spectrum blocks in cognitive radio networks," in Proceedings of the 8th ACM International Symposium on Mobile Ad Hoc Networking and Computing (MobiHoc '07), pp. 130-139, Montreal, Canada, September 2007.

[32] M. Parsapoor and U. Bilstrup, "Imperialist competitive algorithm for DSA in cognitive radio networks," in Proceedings of the 8th International Conference on Wireless Communications, Networking and Mobile Computing (WiCOM '12), pp. 1-4, IEEE, Shanghai, China, September 2012.

[33] M. Manzano, F. Espinosa, Á. M. Bravo, D. García, A. Gardel, and I. Bravo, "Medium access control based on a non cooperative cognitive radio for platooning communications," in Proceedings of the IEEE Intelligent Vehicles Symposium (IV '12), pp. 408-413, June 2012.

[34] S. Eichler, "Performance evaluation of the IEEE 802.11p WAVE communication standard," in Proceedings of the 66th IEEE Vehicular Technology Conference (VTC '07), pp. 2199-2203, October 2007.

[35] S. Kumar, V. S. Raghavan, and J. Deng, "Medium access control protocols for ad hoc wireless networks: a survey," Ad Hoc Networks, vol. 4, no. 3, pp. 326-358, 2006.

[36] J. Mitola III and G. Q. Maguire Jr., "Cognitive radio: making software radios more personal," IEEE Personal Communications, vol. 6, no. 4, pp. 13-18, 1999.

[37] M. di Felice, K. R. Chowdhury, and L. Bononi, "Analyzing the potential of cooperative cognitive radio technology on intervehicle communication," in Proceedings of the IFIP Wireless Days (WD '10), October 2010.

[38] S.-E. Chung, J. Yoo, and C.-K. Kim, "A cognitive MAC for VANET based on the WAVE systems," in Proceedings of the 11th International Conference on (ICACT '09), pp. 41-46, Dublin, Ireland, February 2009.

[39] S. Gräfling, P. Mähönen, and J. Riihijärvi, "Performance evaluation of IEEE 1609 WAVE and IEEE 802.11p for vehicular communications," in Proceedings of the 2nd International Conference on Ubiquitous and Future Networks (ICUFN '10), pp. 344-348, June 2010.

[40] http://www.geintra-uah.org/idi/sofware-abierto/sofwareabierto.

[41] L. Roberts, "Aloha packet system with and without slots and capture," ACM SIGCOMM Computer Communication Review, vol. 5, no. 2, pp. 28-42, 1975.

[42] F. Borgonovo, A. Capone, M. Cesana, and L. Fratta, RRALOHA, a Reliable R-ALOHA Broadcast Channel for Ad-Hoc Inter-Vehicle Communication Networks, Dipart Elettronica e Informazione, Politec di Milano, 2002, ftp://ftp.dei.polimi.it/ outgoing/Flaminio.Borgonovo/PUBBC/2002medhoc.pdf. 


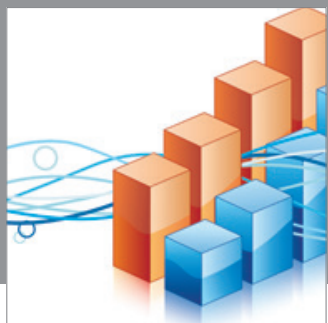

Advances in

Operations Research

mansans

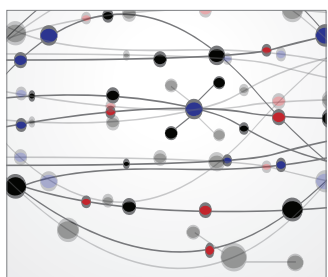

The Scientific World Journal
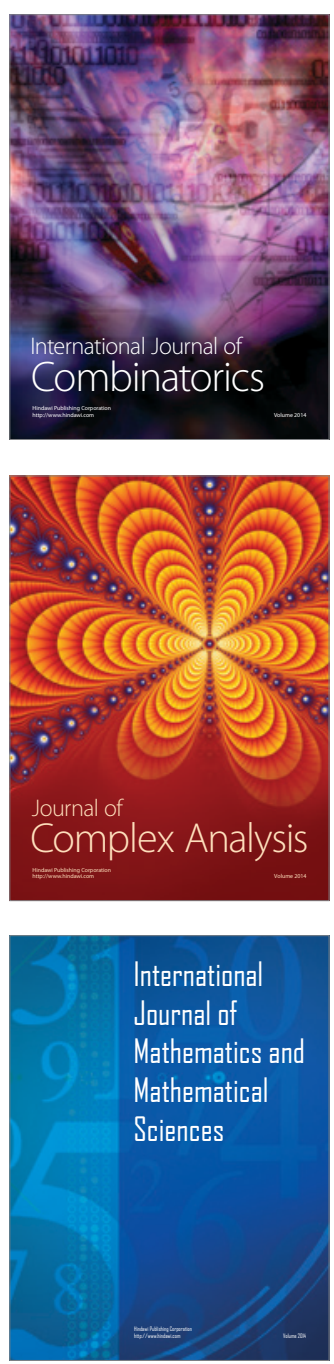
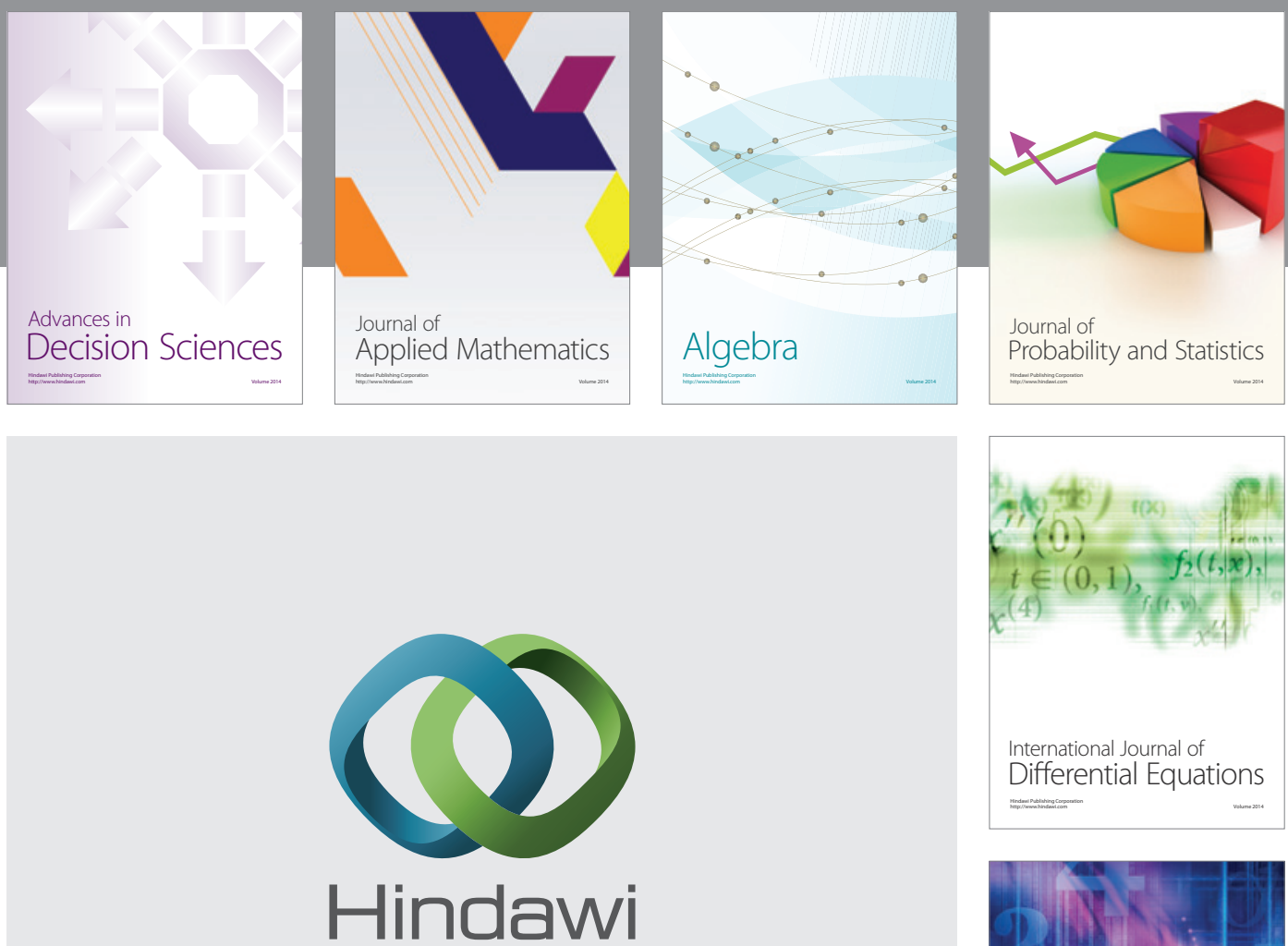

Submit your manuscripts at http://www.hindawi.com
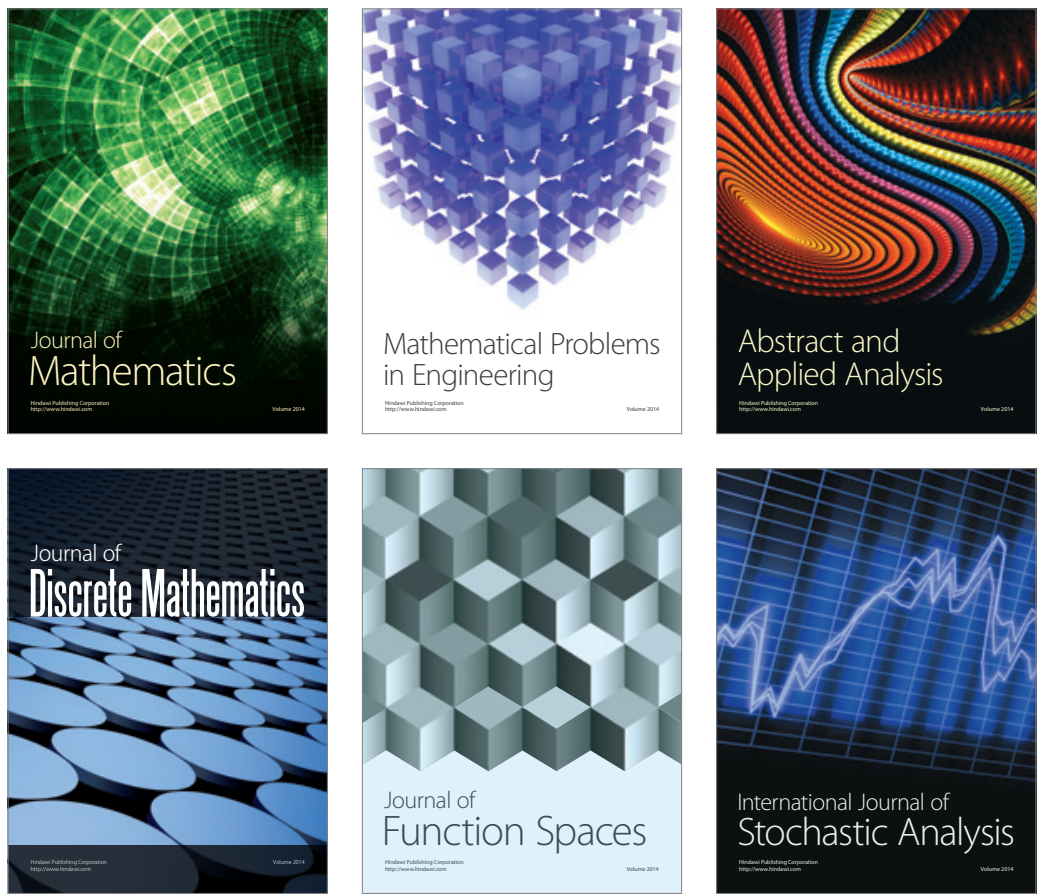

Journal of

Function Spaces

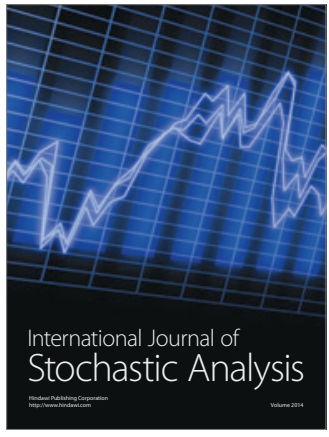

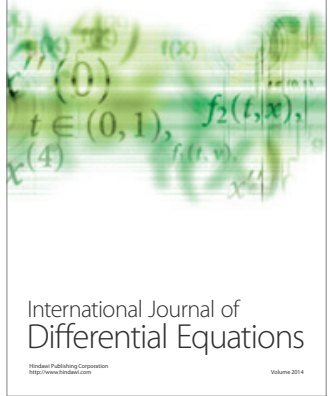
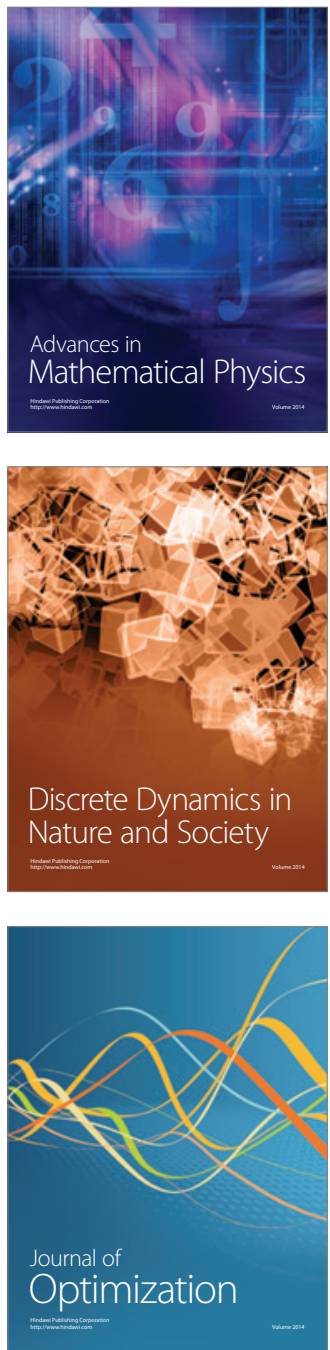